\title{
A Extensão Universitária e a Promoção da Saúde no Brasil: Revisão Sistemática
}

The University Extension and the health Promotion in Brazil: Systematic Review

La Extensión Universitaria y la Promoción de la Salud en Brasil: Revisión Sistemática

Josineide Francisco Sampaio ${ }^{1}$

Cristiana Carina de Barros Lima Dantas Bittencourt ${ }^{2}$

Vanessa Fernandes de Almeida Porto ${ }^{3}$

Jairo Calado Cavalcante ${ }^{4}$

Mércia Lamenha Medeiros ${ }^{5}$

\section{Resumo}

Objetivo: Descrever um estado da arte da publicação científica e refletir sobre a contribuição da extensão universitária para a implementação e desenvolvimento de ações de promoção em saúde no Brasil. Método: Realizouse uma revisão sistemática de literatura, com busca na Biblioteca Virtual em Saúde, baseada nos estudos publicados entre o período de 2007 a 2016, a partir dos descritores Extensão Comunitária, Promoção da Saúde, Promoção em
Saúde, Extensão Universitária e Educação em Saúde. Os dados foram apresentados sob a forma de valores absolutos e relativos, construídos Intervalos de Confiança Exatos pelo programa Epidat versão 3.1 e executada a Análise de Regressão e construção de gráfico através do Microsoft Excel ${ }^{\circledR}$ versão 2010. Resultados: Obteve-se como resultado, 05 publicações relacionadas ao tema, das quais foram construídos dois conjuntos de informações com suas respectivas referências e a descrição quanto ao tipo

${ }^{1}$ Doutora em Saúde Pública pela ENSP/FIOCRUZ, Prof. a da Faculdade de Medicina - FAMED/UFAL. Autor correspondente: Rua José de Alencar, 102, Farol. 575051-565. Maceió, Al, Brasil. E-mail: josineide.sampaio@famed.ufal.br

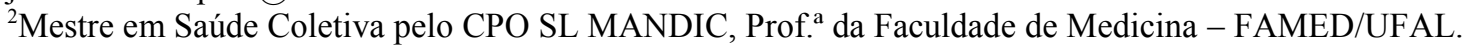

${ }^{3}$ Mestre em Ensino na Saúde pela FAMED/UFAL, Prof. a de Fonoaudiologia - UNCISAL.

${ }^{4}$ Esp. em Epidemiologia ENSP/FIOCRUZ, Prof. da Faculdade de Medicina - FAMED/UFAL.

${ }^{5}$ Doutora em Ciências Aplicadas à Pediatria pela UNIFESP, Prof. da Faculdade de Medicina FAMED/UFAL.

Recebido: Ago./2018 - Aceito: Dez./2018. 
de estudo, resultados e conclusões, e outro que descreve as principais contribuições da extensão universitária para a promoção da saúde. Ainda foram reconhecidas as ações de extensão desenvolvidas, regiões e estados onde as mesmas se realizaram. Conclusão: assinala-se a importância do desenvolvimento das atividades de extensão e as suas principais contribuições para a promoção de saúde sob diversas perspectivas e cenários.

\section{Descritores: Relações Comunidade- Instituição; Educação em Saúde; Promoção da Saúde.}

\section{Abstract}

Objective: Describe a state of the art of scientific publication and reflect on the contribution of university extension to the implementation and development of health promotion actions in Brazil. Method: A systematic review of the literature was conducted, by searching for published studies in the Virtual Health Library between 2007 and 2016, using the keywords (in Portuguese) Community Extension, Health Promotion, Health Promotion, University Extension and Health Education. The data was organized with multivariate values, with constructed
Confidence Intervals by using the software program Epidat version 3.1 and Regression Analysis and graph construction through Microsoft Excel ${ }^{\circledR}$ version 2010. Results: Obtained as results 5 publications related to the subject were gathered, organized in two tables that present the reference of the article, its description as to the type of study, results and conclusions, and that identify the main contributions of university extension to health promotion. Moreover, developed extension actions were identified as well as, regions and states where such actions happened. Conclusions: The main findings are the manifolds of extension on the promotion of health as well as the central role of extension activities to this context.

\section{Descriptors: Community-Institutional} Relations; Health Education; Health Promotion.

\section{Resumen}

Objetivos: Describir un estado de la publicación científica y refletir acerca de la contribución de la extensión universitaria para la aplicación y el desarrollo de acciones de promoción de la salud en Brasil. Métodos: Se realizó una revisión sistemática de la 
literatura, para buscar en la Biblioteca

Virtual en Salud, estudios publicados entre 2007 y 2016, y que tuvian las palabras claves Promoción de la Salud, Extensión en la Universidad y Educación en Salud. Los datos fueron presentados en forma de valores absolutos y relativos, construidos Intervalos de Confianza Exactos por el programa Epidat versión 3.1 y ejecutada el Análisis de Regresión y construcción de gráfico a través de Microsoft Excel @ versión 2010. Resultados: Fueran seleccionados 05 estudios relacionados con el tema, y organizados en das tablas con las referencias, una descripción del tipo de estudio, resultados y conclusiones, $e$ identificar las principales aportaciones de extensión universitaria para la promoción de la salud. También se identificaron las acciones de extensión desarrolladas, regiones y estados donde las mismas se realizaron. Conclusiones: Se identifica la importancia del proceso de desarrollo de las actividades de extensión $y$ sus principales contribuciones a la promoción de la salud desde diversas perspectivas y escenarios.

Descriptores: Relaciones ComunidadInstitución; Educación en Salud; Promoción de la Salud.

\section{Introdução}

A partir dos anos 2009 e 2010, a Extensão Universitária tornou-se o instrumento de inter-relação da Universidade com a sociedade, de democratização do conhecimento e reprodução desse conhecimento por meio da troca de saberes com as comunidades. A extensão atua como um processo interdisciplinar, cultural, educativo, científico, de interação dialógica, no qual ocorre uma via de mão-dupla entre sociedade e universidade ${ }^{(1)}$.

Atualmente $\mathrm{o}$ processo de mudança da sociedade, também acarretou transformações na saúde da população. Diante disto, a Política Nacional de Promoção da Saúde visa estratégias para o enfrentamento dos desafios de produção da saúde num cenário sócio histórico cada vez mais complexo e que demanda a reflexão e qualificação contínua das práticas sanitárias e do sistema de saúde ${ }^{(2)}$.

Desta forma, a promoção da saúde atua como uma das ferramentas de produção de saúde, quer dizer, como um modo de pensar e de operar articulado às demais políticas e tecnologias desenvolvidas no sistema de saúde brasileiro, colaborando na construção de 
ações que possibilitam responder às necessidades sociais em saúde ${ }^{(2)}$.

No Brasil, os princípios da promoção de saúde vêm sendo utilizados na reestruturação do sistema de saúde público, com base nas premissas do Sistema Único de Saúde (SUS). Ressaltam-se também que nesta reestruturação, as propostas de promoção atribuem liberdade de escolha ao indivíduo usuário do sistema público, vinculando-o à responsabilidade social $^{(3)}$.

A promoção de saúde deve ser um processo que vise capacitar os sujeitos e comunidades para desenvolverem seus potenciais de saúde, ou seja, possibilitando oportunidades de conhecimento e controle sobre fatores determinantes. Assim sendo, realizar um projeto de extensão com a população, tendo como referência a promoção de saúde, é uma estratégia para atender às diretrizes das políticas ministeriais $^{(4)}$.

A função da Extensão na universidade é promover o aprendizado que transpasse os espaços acadêmicos e possibilite a interlocução entre $\mathrm{o}$ conhecimento científico e o popular, viabilizando a relação transformadora entre universidade e sociedade ${ }^{(5)}$.

Além disso, a extensão proporciona que o estudante enfrente situações reais, desenvolvendo sua capacidade de pensar sobre diferentes experiências vivenciadas em grupo, bem como seu amadurecimento profissional e pessoal $^{(6)}$.

Acredita-se que, por inserir os discentes de maneira ativa e participativa no contexto social brasileiro, a extensão tem contribuído para uma formação acadêmica mais humanizada, generalista e crítica aos acadêmicos envolvidos, repleta de reflexividade e atentas à realidade social $^{(7)}$.

A partir de tais elementos, objetivou-se nesta revisão sistemática de literatura, descrever um estado da arte da publicação científica e refletir sobre a contribuição da extensão universitária para a implementação e desenvolvimento de ações de promoção em saúde no Brasil.

\section{Método}

Para o levantamento da produção científica desta revisão sistemática, foi realizada busca na Biblioteca Virtual em Saúde (BVS), a qual integra importantes bases de dados nacionais e internacionais de publicações científicas na área da saúde. Inicialmente, buscaram-se os descritores Extensão Comunitária, Promoção da 
Saúde, Promoção em Saúde, Extensão Universitária e Educação em Saúde, inseridos nos Descritores em Ciências da Saúde (DeCS). Apesar de não constar neste, observou-se a necessidade de inserir o descritor "Extensão Universitária", por possibilitar encontrar maior quantidade de publicações. Ainda na delimitação do estudo, foram selecionados artigos publicados entre o período de 2007 a 2016. Inicialmente, foi realizada a leitura dos títulos e resumos dos textos selecionados, sendo então analisados o total de 5 artigos, visto que correspondiam ao objetivo da pesquisa e estavam disponíveis na íntegra na base de dados.

Para a sistematização e análise dos dados, usaram-se dois conjuntos de informações: no primeiro foi apresentada a referência dos artigos, a sua descrição quanto ao tipo de estudo, resultados e conclusões; e no segundo foram distinguidas as principais contribuições da extensão universitária para a promoção da saúde.

Tendo como base os relatos dos artigos, foram agrupadas as ações de extensão desenvolvidas, e ainda, as regiões e estados onde as mesmas se realizaram.

Os dados foram apresentados sob a forma de valores absolutos e relativos.
Foram construídos Intervalos de Confiança Exatos pelo programa Epidat versão 3.1. A Análise de Regressão e a construção de gráfico foi executada pelo Microsoft Excel ${ }^{\circledR}$ versão 2010.

\section{Resultados e Discussão}

Dos 5.032 artigos encontrados, foram selecionados 31 e analisados apenas 5, o restante foi descartado por duplicações ou não corresponderem aos objetivos da pesquisa. Com o termo Extensão Comunitária combinada com Promoção da Saúde ou Promoção em Saúde ou Educação em Saúde foram encontrados $4.788(95,2 \%)$ dos artigos, os resultados restantes resultaram da busca do termo Extensão Universitária combinada com os termos citados na combinação anterior.

Em relação à distribuição anual das publicações, observou-se que nos anos iniciais e finais do período em estudo, nenhuma publicação foi analisada e que a associação com os anos foi negativa e muito fraca $\mathrm{R}^{2}=$ 0,0061, sendo 2012 o único ano em que foram analisados 2 artigos.

Para melhor compreensão do tema, buscou-se encontrar a distribuição e o tipo de ações extensionistas desenvolvidas no âmbito da promoção da saúde, por observar que as 
publicações analisadas correspondem a estudos de caso, tendo como objeto experiências singulares como indica a tabela 1 .

Observou-se que foi uma diversidade de ações e que o grupo de conversa foi o mais utilizado como ação estratégica. Também se observou que os intervalos de confiança exatos foram todos significativos, embora muito largo, considerando a baixa precisão na estimativa do valor amostral.

Tabela 1 - Distribuição Segundo Ações Relatadas nos Artigos

\begin{tabular}{|c|c|c|c|c|}
\hline \multirow{2}{*}{ Ações realizadas } & \multirow{2}{*}{$\mathbf{n}$} & \multirow{2}{*}{$\%$} & \multicolumn{2}{|c|}{ IC95\% } \\
\hline & & & Linf & Lsup \\
\hline Grupo de conversa & 2 & 40,0 & 5,3 & 85,3 \\
\hline Grupo operativo comunitário & 1 & 20,0 & 0,5 & 71,5 \\
\hline Oficina & 1 & 20,0 & 0,5 & 71,5 \\
\hline $\begin{array}{l}\text { Visita domiciliar e reuniões } \\
\text { comunitárias }\end{array}$ & 1 & 20,0 & 0,5 & 71,5 \\
\hline Total & 5 & 100,0 & & \\
\hline
\end{tabular}

Também foi descrito a localidade onde as ações apresentadas nos artigos foram desenvolvidas e a sua distribuição por região e estado (Tabela

2).

Tabela 2 - Distribuição dos Estudos Segundo Regiões e Estados

\begin{tabular}{llcccc}
\hline Regiões & Estados & n & \% & \multicolumn{2}{c}{ IC95\% } \\
\hline Sudeste & São Paulo & 2 & 40,0 & 5,27 & 85,34 \\
Nordeste & Paraíba & 1 & 20,0 & 0,51 & 71,54 \\
Sul & Paraná & 1 & 20,0 & 0,51 & 71,54 \\
Centro-Oeste & Mato Grosso do Sul & 1 & 20,0 & 0,51 & 71,54 \\
Total & & 5 & 100,0 & & \\
\hline
\end{tabular}

Observou-se que o estado de São Paulo conta com o maior número de ações analisadas, e igualmente a tabela anterior, os intervalos de confiança exatos foram todos significativos, embora muito largo, considerando a baixa precisão na estimativa do valor amostral.
A partir da análise das publicações, obteve-se um levantamento dos tipos de estudo e dos resultados e conclusões sobre a extensão universitária e sua contribuição para a promoção da saúde:

1 - Estudo de Combinato ${ }^{(4)}$ : 
Estudo de Caso com Abordagem Qualitativa. Os resultados e conclusões foram: Oficinas de Estimulação, voltadas para o brincar, constitui-se em um espaço no qual as crianças pudessem ter contato intenso com recursos lúdicos variados de maneira dirigida. Promoveu-se a integração entre as crianças; formação de vínculos entre elas; acolhimento da espontaneidade; desenvolvimento de comportamentos sociais mais adequados e cooperativos; troca tanto no sentido relacional, quanto no cultural; houve lugar tanto para o brincar dirigido quanto para $\mathrm{o}$ espontâneo. É fundamental que o direito de brincar esteja associado a uma educação formal de boa qualidade, outros serviços de saúde pública, acesso à cultura, ao lazer, entre outros aspectos de direito de todos os cidadãos, para que haja a promoção de saúde infantil de maneira efetiva.

\section{2 - Estudo de Cruz, Vieira; Massa,} Araújo, Vasconcelos ${ }^{(7)}$ :

Relato de Experiência. Os resultados e conclusões foram: As atividades em grupo consistiram em um espaço privilegiado para a constituição de redes de apoio, estabelecimento e ampliação de vínculos afetivos, reflexão e conscientização das determinações do processo saúde-doença, organização e mobilização para o efetivo controle social, além de ser um espaço de ensino-aprendizagem, orientação, intervenção e educação em saúde.

\section{3 - Estudo de Imbrizi $^{(8)}$ :}

Estudo de Caso com Abordagem Qualitativa. Os resultados e conclusões foram: A participação popular constitui uma força social capaz de elencar prioridades e influir nos serviços públicos de saúde. Problemas cotidianos da USF podem ser resolvidos pela negociação por meio de diálogo, da criação de vínculos e de parcerias. A universidade pode contribuir para o fortalecimento da gestão participativa como espaço de formação e informação relacionado ao controle social. E a vivência em processos de participação popular contribui para uma formação humanística e imprescindível para o profissional do SUS.

\section{4 - Estudo de Kracker, Kolesnikovas}

e Kato ${ }^{(3)}$ :

Estudo de Caso com Abordagem Qualitativa. Os resultados e conclusões foram: Necessidade de apoio das 
equipes de saúde ao trabalho dos ACS, por meio de reuniões amplas sobre a organização do trabalho na unidade, dando prioridade às visitas domiciliares e não as tarefas administrativas. Necessidade de reconhecimento do trabalho dos ACS através da melhoria salarial e plano de carreira. Participação ativa de discussões em conjunto sobre os diversos conhecimentos inerentes ao processo saúde, doença e cuidado da população. Necessidade de cursos de capacitação e educação permanente relacionada às políticas de saúde e sociais e de como implanta-las na comunidade.

\section{5 - Estudo de Nunes, Montibeller,}

Oliveira, Arrabaca, Theiss ${ }^{(5)}$ :
Estudo de Caso com Abordagem Qualitativa. Os resultados e conclusões foram: As atividades desenvolvidas proporcionaram a cada integrante a autorreflexão, o cuidado de si, e a elevação da autoestima, pois todas as pessoas que participaram do grupo apresentaram melhoras significativas em suas vidas.

A partir da análise dos resultados e conclusões das publicações foram reconhecidas as principais contribuições da extensão universitária para a promoção da saúde, apresentadas a seguir no quadro 1.

\section{Quadro 1 - Principais Contribuições da Extensão para Promoção da Saúde}

\begin{tabular}{|l|c|}
\hline \multicolumn{1}{|c|}{ Contribuições } & \multicolumn{1}{c|}{ Código } \\
\hline $\begin{array}{l}\text { Constituição e ampliação de redes de interação } \\
\text { e de apoio }\end{array}$ & $\begin{array}{c}\text { Artigo }^{4}, \mathrm{Artigo}^{7} \\
\mathrm{Artigo}^{8}, \mathrm{Artigo}^{7}\end{array}$ \\
\hline $\begin{array}{l}\text { Fortalecimento da gestão participativa e do } \\
\text { controle social }\end{array}$ & $\mathrm{Artigo}^{7}, \mathrm{Artigo}^{8}, \mathrm{Artigo}^{3}$ \\
\hline Integração social e vínculos afetivos & $\mathrm{Artigo}^{4}, \mathrm{Artigo}^{7}, \mathrm{Artigo}^{8}$ \\
\hline $\begin{array}{l}\text { Identificação das demandas organizacionais } \\
\text { dos serviços e dos profissionais de saúde }\end{array}$ & $\mathrm{Artigo}^{8}, \mathrm{Artigo}^{3}$ \\
\hline $\begin{array}{l}\text { Conscientização das determinações do } \\
\text { processo saúde-doença e cuidado de si }\end{array}$ & Artigo $^{7}, \mathrm{Artigo}^{5}$ \\
\hline Formação humanista & Artigo $^{8}$ \\
\hline
\end{tabular}

Pode-se observar que entre as principais contribuições da extensão para a promoção de saúde, destacou-se a criação e ampliação das redes de interação e de apoio junto aos usuários, profissionais e serviços sociais e de saúde; o fortalecimento da gestão participativa e do controle social; a 
promoção da integração social e dos vínculos afetivos; a identificação das demandas organizacionais dos serviços e dos profissionais de saúde; a conscientização sobre as determinações do processo saúde-doença e o cuidado de si; e o desenvolvimento de uma formação humanista.

Percebe-se que estas contribuições também estão relacionadas às dimensões e aos princípios das ações de promoção de saúde apresentadas por Kracker, Kolesnikovas e Kato (2009), considerando-se que para a elaboração e implementação de políticas públicas saudáveis, realizou-se o fortalecimento da gestão participativa e do controle social, a identificação das demandas organizacionais dos serviços e dos profissionais de saúde e se promoveu uma formação humanista. Quanto à dimensão criação de ambientes favoráveis à saúde, a mesma foi desenvolvida por meio da integração social e de vínculos afetivos, identificação das demandas organizacionais dos serviços e dos

\section{Conclusão}

Diante do exposto, observa-se que o termo Extensão Universitária, apesar profissionais de saúde e formação humanista. Em relação ao reforço de ação comunitária, foi desenvolvido com a constituição e ampliação de redes de interação e de apoio, fortalecimento da gestão participativa e controle social, integração social e vínculos afetivos, identificação das demandas organizacionais dos serviços e dos profissionais de saúde, conscientização das determinações do processo saúdedoença e cuidado de si. Para o desenvolvimento de habilidades pessoais foram estimulados a integração social e os vínculos afetivos, a conscientização das determinações do processo saúde-doença e o cuidado de si, além de uma formação humanista. Quanto à dimensão reorientação dos serviços de saúde, ocorreu a partir da constituição e ampliação de redes de interação e de apoio, fortalecimento da gestão participativa e controle social, conscientização das determinações do processo saúde-doença e cuidado de si e formação humanista.

de não estar incluso nos Descritores em Ciências da Saúde (DeCS), apresentouse como um termo importante na busca relacionada à temática. Também se assinala a importância do desenvolvimento das atividades de extensão e as suas principais 
contribuições para a promoção de saúde sob diversas perspectivas e cenários.

A partir da análise dos desenhos de estudos das publicações, observa-se que há um número reduzido de trabalhos publicados que versem sobre a temática, sendo em sua maioria estudos de caso e relatos de experiência, que embora sejam relevantes, evidenciam a necessidade de estudos que possibilitem mensurar a sua dimensão e o seu alcance, bem como, o fortalecimento da pesquisa no âmbito da extensão universitária no país.

\section{Referências}

1- Ministério da Educação (BR). Fórum de Pró-Reitores de Extensão das Universidades Públicas Brasileiras e SESu/MEC. Plano Nacional de Extensão Universitária. Brasília: Ministério da Educação; 2012.

2- Ministério da Saúde (BR). Secretaria de Vigilância em Saúde. Secretaria de Atenção à Saúde. Política Nacional de Promoção da Saúde. Brasília: Ministério da Saúde; 2006.

3- Kracker CK, Kolesnikovas IH, Kato, RAF. Oficina de Estimulação Infantil: Uma prática de promoção de saúde coletiva em um equipamento da rede substitutiva de saúde mental. Psicologia (São Paulo) [periódico na internet]. 2009 [citado 2018 jan. 31]; 18(1): 113-31. Disponível em: https://revistas.pucsp.br/index.php/psic orevista/article/view/2965.

4- Combinato DS, et al. "Grupos de Conversa": Saúde da pessoa Idosa na Estratégia Saúde da Família. Psicologia (Minas Gerais) [periódico na internet]. 2010 [citado 2018 jan. 31]; 22(3): 558-68. Disponível em:
http://www.scielo.br/scielo.php?pid=S 0102-71822010000300016\&script $=$ sci abstract\&tlng $=$ pt.

5- Nunes MRM, Montibeller C, Oliveira K, Arrabaca, RCB, Theiss SMMB. Autoestima e saúde mental: Relato de experiência de um projeto de extensão. Psicologia (Curitiba) [periódico na internet]. 2013 [citado 2018 fev. 15]; 31(73): 283-89. Disponível em: https://periodicos.puc pr.br/index.php/psicologiaargumento/a rticle/view/20487/19741.

6- Porto VFA, Bittencourt CCBLD, Sampaio JF. Uma revisão de literatura sobre as contribuições da extensão universitária para a formação profissional em saúde. In: Sampaio JF, et al. A extensão universitária na formação em saúde. Maceió: Edufal; 2015;(1):13-2.

7- Cruz PJSC, Vieira SCR, Massa NM, Araújo TAM, Vasconcelos ACCPA. Desafios para a Participação Popular em Saúde: reflexões a partir da educação popular na construção de conselho local de saúde em comunidades de João Pessoa, PB. Saúde (São Paulo) [periódico na internet]. 2012 [citado 2018 fev. 23]; 21(4): 1087-100. Disponível em: http://www.scielo.br/scielo.php?pid=S 0104-12902012000400025\&script $=$ sci _abstract\&tlng $=$ pt.

8- Imbrizi, JM. et al. Condições de trabalho na Estratégia de Saúde da Família: relato de experiência de extensão universitária com agentes comunitários de saúde. Psicologia (São Paulo) [periódico na internet]. 2012 [citado 2018 mar. 13]; 15(1): 153-69. Disponível em: http://pepsic.bvsalud. org/scielo.php?script=sci_abstract\&pid $=$ S1516-37172012000100011. 